\title{
A Pure Signal-Based Stiffness Estimation for VSA Devices
}

Fabrizio Flacco

\begin{abstract}
The capability of controlling both the position/torque and the stiffness of the joints is the main feature of the next generation of robots based on Variable Stiffness Actuators (VSA). For the purpose of accurate control, recent works have pointed out that is not possible to rely completely on analytical models of the stiffness characteristics of the transmissions/joints and that an on-line estimation of stiffness is often mandatory. Building on our previous results, we present a new method to estimate the stiffness based only on inputoutput signals, without any knowledge of motor parameters nor the need of joint torque sensing. In addition, a Recursive Least Squares method based on a QR decomposition (QR-RLS) is used, which is very robust to poor excitation conditions. In order to deal more efficiently with noisy signals, a combination of two filtering actions is also considered, with a causal Kinematic Kalman Filter (KKF) and a non-causal SavitzkyGolay (SG) filter. Simulation results and comparison with two other state-of-the-art stiffness estimators are presented.
\end{abstract}

\section{INTRODUCTION}

Stiffness of a flexible transmission is defined as the variation of the transmitted torque with respect to a variation of its deformation. It represents the opposition of a compliant transmission to any factor causing the deflection, typically the actuation torque applied by a motor or an external torque applied to the driven load.

Variable Stiffness Actuators (VSA) consist of two motors and one or more nonlinear flexible transmissions, arranged so as to allow separate control of output motion and device stiffness. No matter if the objective of using VSA joints is safety [1], [2], obtaining explosive motions [3], [4], or optimizing some performance criteria [5], [6], for accurate control purposes the current value of the stiffness is always needed. However, there are no sensors available for a direct measure of the stiffness, in particular that can be used on line (i.e., during dynamic robot operation). The device stiffness is usually computed from position and/or joint torque sensor data, based on a nominal mathematical model. This may be critical for VSAs since $i$ ) the stiffness characteristic profiles are intrinsically nonlinear, $i i$ ) the mathematical model can be a complex function of the deformation of the transmissions, subject to kinematic and dynamic uncertainties, and iii) the stiffness is a variable parameter that should be set explicitly under control.

For these reasons. an accurate and reliable estimate of the stiffness of flexible transmissions is necessary, and a number of different solutions have been proposed so far.

The authors are with the Dipartimento di Ingegneria Informatica, Automatica e Gestionale, Sapienza Università di Roma, Via Ariosto 25, 00185 Roma, Italy (\{fflacco,deluca $\} @$ diag.uniroma1.it). This work is supported by the European Commission, within the FP7 ICT-287513 SAPHARI project (www.saphari.eu)
Alessandro De Luca

In [7], a dynamic observer was introduced that achieves ultimately bounded error in stiffness estimation. Knowledge of the applied external torque and of its time derivative is assumed and (multiple) numerical differentiation of the measured position is needed. In [8], this approach was developed and complemented by an Extended Kalman Filter (EKF) in order to estimate simultaneously the transmission stiffness as well as the (link) inertia and damping. The need of numerical differentiation of the joint torque measured by an extra sensor, together with the presence of an interaction loop, led to high sensitivity to signal noise. In [9], drawbacks were partly alleviated by resorting to a parametric model of the torque/deformation characteristic. This idea, which was introduced independently also in [10] and is reprised in the present paper, eliminates the need of multiple numerical differentiation of signals. However, the stiffness estimator in [9] still relies on the use of a joint torque sensor. In [11]. an optoelectronic sensor is used to estimate the instantaneous elastic energy stored in the transmission, which is provided then as input to an adaptive algorithm for estimating the stiffness.

All the above methods, including those based on link side measurements, are invasive, because they require extra sensors mounted on the transmission. On the other hand, a benefit arises when considering the same stiffness estimation problem but seen from the motor side. Following this line of thought, we have proposed in [10] an initial set of stiffness estimators for antagonistic VSAs that does not need joint torque sensing and is completely independent from gravity or other external forces (including those due to dynamic interactions in a multi-link robot). The algorithm was structured in two phases, an estimation of the flexibility torque using a residual-based method followed then by different regressors. This two-phase approach was used also in [12], where the second phase was replaced by a polynomial fitting based on Recursive Least Squares (RLS). This idea was revised and extended to serial VSAs in [13], [14]. A two-phase approach working on the motor side was used also in [15]. While the second phase relies on similar RLS techniques, the first phase has been replaced with a series of FIR filters designed using the theory of operational calculus. The result is a stiffness estimator with a high robustness w.r.t. sensor noise.

In this paper, we present an improved on-line stiffness estimation method that relies only on input-output signals. Its main feature is that stiffness can now be estimated even without any knowledge of the motor parameters, relaxing the only model information still needed by our previous approach. By integration of the motor equation, these parameters become just extra terms in the estimation process. 
The original RLS method is replaced by a more robust RLS algorithm based on QR decomposition (QR-RLS). Moreover, a new filtering action based on the combination of a causal Kinematic Kalman Filter (KKF) and a non-causal SavitzkyGolay (SG) filter allows to obtain good results even in case of large sensor and/or actuator noise.

The paper is organized as follows. The model of an antagonistic VSA is recalled in Sect II. Sections III and IV present the complete approach. Simulation results are reported in Sect. $\mathrm{V}$ for the case of ideal signals. Noise filtering actions are described in Sect. VI, and simulations with realistic signal models are used in Sect. VII to illustrate the actual performance of the estimation method.

\section{BACKGROUND}

Flexible transmissions are characterized by elements that allow a deformation $\phi=q-\theta$ between the motor angle $\theta$ and the link angle $q$. A smooth potential function $U_{e}(\phi) \geq 0$ is associated to the deformation $\phi$, with $U_{e}(\phi)=0$ iff $\phi=$ 0 . The flexibility torque across the transmission is $\tau_{e}(\phi)=$ $\partial U_{e}(\phi) / \partial \phi$. The stiffness of the transmission can be defined as the variation rate of the flexibility torque $\tau_{e}(\phi)$ w.r.t. the deformation $\phi$,

$$
\sigma(\phi)=\frac{\partial \tau_{e}(\phi)}{\partial q}=\frac{\partial \tau_{e}(\phi)}{\partial \phi}>0
$$

For a single motor driving a rigid link subject to gravity through a (nonlinear) flexible transmission (Fig. 1a), the dynamic model takes the form

$$
\begin{aligned}
M \ddot{q}+D_{q} \dot{q}+\tau_{e}(\phi)+g(q) & =\tau_{\text {ext }} \\
B \ddot{\theta}+D_{\theta} \dot{\theta}-\tau_{e}(\phi) & =\tau,
\end{aligned}
$$

where $M>0$ and $B>0$ are the link and motor inertias, $D_{q} \geq 0$ and $D_{\theta} \geq 0$ are the viscous friction coefficients at the two sides of the transmission, $\tau$ is the control torque on the motor side, and $g(q)$ and $\tau_{\text {ext }}$ are respectively the gravity and any environment/disturbance torques acting on the link.

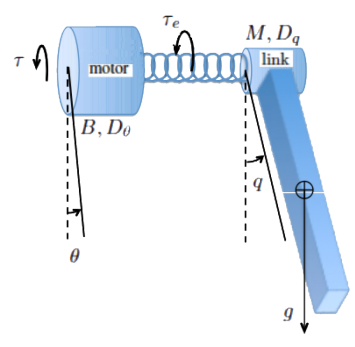

(a)

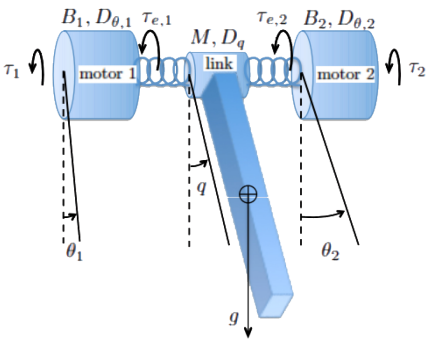

(b)
Fig. 1. Schematic model of a single link driven by a flexible transmission (a) or by a VSA in antagonistic arrangement (b)

An antagonistic VSA (Fig. 1b) is characterized by two motors working in parallel and antagonistically connected to the driven link through nonlinear transmissions. Depending on the realization, the nonlinearity of the deformation/torque characteristics of the transmissions results either from the use of nonlinear (e.g., cubic or exponential) springs or form the arrangement of linear springs in a nonlinear kinematic mechanism [16], [17]. Representative devices in this class are the biologically inspired VSA [18] and the VSA-II [2].

The two motor-transmission units are modeled with two similar equations of the form (3), where each transmission undergoes a deformation $\phi_{i}=q-\theta_{i}$, for $i=1,2$. The dynamics of an antagonistic VSA is thus

$$
\begin{aligned}
M \ddot{q}+D_{q} \dot{q}+\tau_{e, t}(\phi)+g(q) & =\tau_{\text {ext }} \\
B_{i} \ddot{\theta}_{i}+D_{\theta, i} \dot{\theta}_{i}-\tau_{e, i}\left(\phi_{i}\right) & =\tau_{i}, \quad i=1,2 .
\end{aligned}
$$

In this case, the (total) flexibility torque transmitted to the driven link and the associated device stiffness are given by

$$
\tau_{e, t}=\tau_{e, 1}\left(\phi_{1}\right)+\tau_{e, 2}\left(\phi_{2}\right)
$$

and

$$
\sigma_{t}(\phi)=\sigma_{1}\left(\phi_{1}\right)+\sigma_{2}\left(\phi_{2}\right)
$$

with $\phi=\left(\phi_{1} \phi_{2}\right)^{T}$ and where

$$
\sigma_{i}\left(\phi_{i}\right)=\frac{\partial \tau_{e, i}\left(\phi_{i}\right)}{\partial \phi_{i}}>0, \quad i=1,2,
$$

are the local stiffnesses of the two transmissions. We stress the separability of the functions (6) and (7), whereas in general $\phi_{1} \neq \phi_{2}$. Most of the times the two motortransmission units are identical (perfect symmetry). However, our developments apply directly to the general case.

\section{Signal-BASEd STIFFNESS Estimator}

The goal is to obtain an estimation of the stiffness based only on input and output signals, and completely independent from model parameters. For this, working on the motor side, the stiffness of each transmission can be estimated separately and the total stiffness is computed then by (7).

Consider the time integration of motor equation (3)

$$
B \dot{\theta}+D_{\theta} \theta-\int_{0}^{t} \tau_{e}(\phi) d s=\int_{0}^{t} \tau d s .
$$

This representation removes the presence of second time derivative of the motor position, which is difficult to estimate especially in presence of sensor noise. The flexibility torque $\tau_{e}(\phi)$ can be approximated by using a linear regressor of $m$ known polynomials $f_{i}(\phi), i=1 \ldots m$, as

$$
\tau_{e}(\phi) \approx \sum_{i}^{m} \alpha_{i} f_{i}(\phi)
$$

Thus, we rewrite (9) as

$$
B \dot{\theta}+D_{\theta} \theta-\sum_{i}^{m} \alpha_{i} \int_{0}^{t} f_{i}(\phi) d s=\int_{0}^{t} \tau d s .
$$

Assuming that only the input and output signals $\tau, \theta, \phi$, and $\dot{\theta}$ are known, we have to estimate the vector of parameters $\boldsymbol{\alpha}=\left(\begin{array}{llll}\alpha_{1} & \alpha_{2} & \ldots & \alpha_{m}\end{array}\right)^{T}$ of the fitting function for the flexible torque, as well as motor inertia $B$ and damping $D_{\theta}$. Note that the motor torque $\tau$ is a known command applied by the user (e.g., imposing a motor current) and should not 
be confused with the flexibility torque $\tau_{e}$ at the joint (the one that a joint torque sensor would measure in dynamic conditions).

Therefore, we would like to determine the parameter vector $\boldsymbol{\xi}=\left(\begin{array}{lll}B & D_{\theta} & \boldsymbol{\alpha}^{T}\end{array}\right)^{T}$ of dimension $n=m+2$ that minimizes the square of the residual error, i.e.,

$$
\left(\int_{0}^{t} \tau d s-\boldsymbol{F}^{T} \boldsymbol{\xi}\right)^{2}
$$

where

$$
\boldsymbol{F}=\left(\begin{array}{lllll}
\dot{\theta} & \theta & \int_{0}^{t} f_{1}(\phi) d s & \ldots & \int_{0}^{t} f_{n}(\phi) d s
\end{array}\right) .
$$

For a data set composed by a matrix $\boldsymbol{A}$, containing $l$ vectors $\boldsymbol{F}_{k}$ sampled at $t=t_{k}=k T_{s}\left(T_{s}\right.$ is the sampling time $)$,

$$
\boldsymbol{A}=\left(\begin{array}{llll}
\boldsymbol{F}_{1}^{T} & \boldsymbol{F}_{2}^{T} & \ldots & \boldsymbol{F}_{l}^{T}
\end{array}\right)^{T}
$$

and by the vector

$$
\boldsymbol{b}=\left(\begin{array}{llll}
\int_{0}^{t_{1}} \tau d s & \int_{0}^{t_{2}} \tau d s & \ldots & \int_{0}^{t_{l}} \tau d s
\end{array}\right)^{T},
$$

the solution that minimizes (12) in batch mode is obtained by pseudo-inversion as

$$
\hat{\boldsymbol{\xi}}=\boldsymbol{A}^{\#} \boldsymbol{b}
$$

From the obtained parameter vector $\hat{\boldsymbol{\xi}}$, we extract the estimated motor inertia $\hat{B}$ and damping $\hat{D}_{\theta}$, while the estimated stiffness is computed analytically as

$$
\hat{\sigma}=\sum_{i}^{m} \hat{\alpha}_{i} \frac{\partial f_{i}(\phi)}{\partial \phi}=\sum_{i}^{m} \hat{\alpha}_{i} g_{i}(\phi) .
$$

No numerical differentiations nor torque sensing are needed.

\section{ON-LINE ROBUST IMPLEMENTATION}

The approach represented by eqs. (14-16) needs to collect a batch of data and is executed thus off-line, implicitly assuming that the parameter vector $\boldsymbol{\xi}$ remains constant over time. This assumption is indeed critical. While the motor inertia $B$ and damping $D_{\theta}$ can be assumed to be constant during a single experiment, they still need to be identified from time to time, which may require the joint to be disassembled for disconnecting the flexible transmission. On the other hand, the parameter vector $\boldsymbol{\alpha}$ cannot be considered constant during an experiment, mainly for two reasons: i) the parameters affecting flexibility of the transmission change slightly, due to temperature drift and stress caused by repetitive movements; ii) the approximation (10) is not able to fit the complete flexibility torque characteristics, and so the parameters in $\boldsymbol{\alpha}$ have to be adapted around different operating points.

For on-line stiffness estimation, use of a Recursive Least Squares (RLS) algorithm was originally proposed in [12], and then used also in [13]-[15]. The drawback of the RLS algorithm is its sensitivity to poor excitation conditions. In such cases, the positive definiteness and symmetry properties of the inverse of the correlation matrix $\left(\boldsymbol{A}^{T} \boldsymbol{A}\right)^{-1}$ used during estimation may get lost, possibly causing the numerical divergence of the process. A simple solution to this problem has been presented in [13], by introducing an additional scalar parameter that needs to be suitably tuned. We use here instead a QR decomposition within the RLS algorithm (QR-RLS), which is able to handle instability issues more in general. Rather than working with the inverse correlation matrix, the QR-RLS algorithm performs a QR decomposition directly on the correlation matrix of the input signal. The outcome is a version of the RLS algorithm that is numerically stable and more robust than the standard one (see [19]).

For least squares minimization, the $\mathrm{QR}$ decomposition is applied to matrix $\boldsymbol{A}$

$$
\boldsymbol{Q} \boldsymbol{A}=\left(\begin{array}{c}
\boldsymbol{R} \\
\boldsymbol{O}_{(l-n) \times n}
\end{array}\right),
$$

where $\boldsymbol{Q}$ is a $l \times l$ orthonormal matrix, $\boldsymbol{R}$ is an $n \times n$ upper triangular matrix, and $\boldsymbol{O}$ is a zero matrix with the given dimensions. Applying the same matrix $Q$ to the data vector $b$ yields

$$
Q b=\left(\begin{array}{c}
p \\
\star
\end{array}\right)
$$

with the $n$-dimensional vector $\boldsymbol{p}$ and a $\star$ representing not relevant values. Thus, the off-line solution (16) is given by

$$
\hat{\xi}=R^{-1} p
$$

The QR-RLS updates the matrix $\boldsymbol{R}_{k}$ at step $k$ (time $t_{k}$ ) using the relation [20]

$$
\widehat{\boldsymbol{Q}}_{k}\left(\begin{array}{c}
\lambda \boldsymbol{R}_{k-1} \\
\boldsymbol{F}_{k}
\end{array}\right)=\left(\begin{array}{c}
\boldsymbol{R}_{k} \\
\mathbf{0}_{1 \times n}
\end{array}\right),
$$

where $\lambda \in[0,1]$ (usually, larger than 0.95 ) is a forgetting factor that discounts old samples, thus increasing the estimator adaptability to non constant parameters. Note that $\widehat{\boldsymbol{Q}}_{k}$ is a $(n+1) \times(n+1)$ matrix, and its dimension does not increase with new data samples. The same orthonormal matrix $\widehat{\boldsymbol{Q}}_{k}$ is used to update $\boldsymbol{p}_{k}$ as

$$
\widehat{\boldsymbol{Q}}_{k}\left(\begin{array}{c}
\lambda \boldsymbol{p}_{k-1} \\
\int_{0}^{t_{k}} \tau d s
\end{array}\right)=\left(\begin{array}{c}
\boldsymbol{p}_{k} \\
\star
\end{array}\right) .
$$

Finally, the on-line parameter estimation is given by

$$
\hat{\boldsymbol{\xi}}_{k}=\boldsymbol{R}_{k}^{-1} \boldsymbol{p}_{k}
$$

and the estimate of the stiffness is obtained using (17) with the current parameter vector $\hat{\boldsymbol{\alpha}}_{k}$.

The QR decomposition (21) can be recursively updated using a series of Givens rotations to zero out non-zero elements on and below the diagonal due to the added row $\boldsymbol{F}_{k}$. It should be noted that an initialization phase of a $n$ samples is needed to set up a complete $\boldsymbol{R}_{n}$ to be used in the recursive estimation. The QR-RLS is not only robust with respect to poor excitation, but is also simple to tune, being $\lambda$ the only parameter to be chosen. 


\section{Results with Ideal InPut-Output Signals}

To show the effectiveness of the proposed method, we present simulations with the VSA-II device developed by the University of Pisa [2]. The nonlinear flexibility torque of the two transmissions of the VSA-II is modeled as

$$
\tau_{e, i}\left(\phi_{i}\right)=2 k_{i} \beta\left(\phi_{i}\right) \frac{\partial \beta\left(\phi_{i}\right)}{\partial \phi_{i}}, \quad i=1,2,
$$

where $k_{i}$ is the (constant) stiffness of the spring in the $i$-th transmission, and

$$
\beta\left(\phi_{i}\right)=\arcsin \left(C_{i} \sin \left(\frac{\phi_{i}}{2}\right)\right)-\frac{\phi_{i}}{2}, \quad i=1,2,
$$

being $C_{i}>1$ a geometric parameter of the 4-bar mechanisms. Indeed, this model is used in the simulations as the ground truth for the stiffness estimator. Due to the antagonistic arrangement, the total flexibility torque acting on the link dynamics is given by the sum in eq. (6). For this reason, and with no loss of generality, we will present just the estimation results for a single transmission if this device.

The VSA-II dynamic model is given by eqs. (4-5), and its nominal parameters were presented in [2]. In particular, the nominal values for the motor parameters were set there to be $B_{N}=7.3[\mathrm{Kg} \cdot \mathrm{m} \cdot \mathrm{mm}]$ and $D_{\theta, N}=1[\mathrm{~N} \cdot \mathrm{mm} \cdot \mathrm{s} / \mathrm{rad}]$. To simulate the slowly time-varying characteristics of the flexible transmission, we have included in the definition of the spring stiffness a small drift over time

$$
k_{1}=k_{1, N}+0.0005 t \quad[\mathrm{~N} \cdot \mathrm{mm} / \mathrm{rad}],
$$

being $k_{1, N}=500[\mathrm{~N} \cdot \mathrm{mm} / \mathrm{rad}]$ the nominal spring stiffness taken from [2]. For comparison purposes, estimation methods that need the availability of motor data will use the nominal data $B_{N}$ and $D_{\theta, N}$ given above. However, in the actual simulated model, we have chosen as ground truth values $B_{A}=7.5[\mathrm{Kg} \cdot \mathrm{m} \cdot \mathrm{mm}]$ and $D_{\theta, A}=0.9[\mathrm{~N} \cdot \mathrm{mm} \cdot \mathrm{s} / \mathrm{rad}]$, which are slightly off the nominal ones mimicking the situation of a small (but realistic) error in the off-line identification phase of the motor dynamics.

Ideal input and output signal have been considered here, i.e., the measured values of $\theta, \dot{\theta}$, and $q$ and the commanded values of $\tau$ coincide with the actual ones. The two motors apply the sinusoidal torques $\tau_{1}(t)=50 \sin 0.1 \pi t$ and $\tau_{2}(t)=50 \sin 2 \pi t \quad[\mathrm{~N} \cdot \mathrm{mm}]$, respectively. The simulation runs with a sampling time $T_{s}=1 \mathrm{~ms}$, starting from $q(0)=$ $\theta_{1}(0)=\theta_{2}(0)=0$ [rad] (lower equilibrium configuration), with the system initially at rest, and moving under gravity. In the fitting function (10), we used $m=7$ polynomial terms $f_{i}(\phi)=\phi^{i}$. The forgetting factor in the QR-RLS algorithm has been set to $\lambda=0.98$.

Figure 2 shows a comparison of stiffness estimation results obtained with the proposed method, with a standard off-line LS fitting, and with the residual/RLS based estimator of [14]. The new method clearly outperforms the other two. The standard LS considers the complete batch of data and is not able to follow time-varying aspects of the flexibility parameters. On the other hand, our method [14] uses the (nominal) motor parameters, and so their imperfect identification is reflected in an error on the estimated stiffness. The proposed method returns also the estimated motor parameters $\hat{B}=7.5135$ $[\mathrm{Kg} \cdot \mathrm{m} \cdot \mathrm{mm}]$ and $\hat{D}_{\theta}=0.9148[\mathrm{~N} \cdot \mathrm{mm} \cdot \mathrm{s} / \mathrm{rad}]$, which are very close to the actual ones.
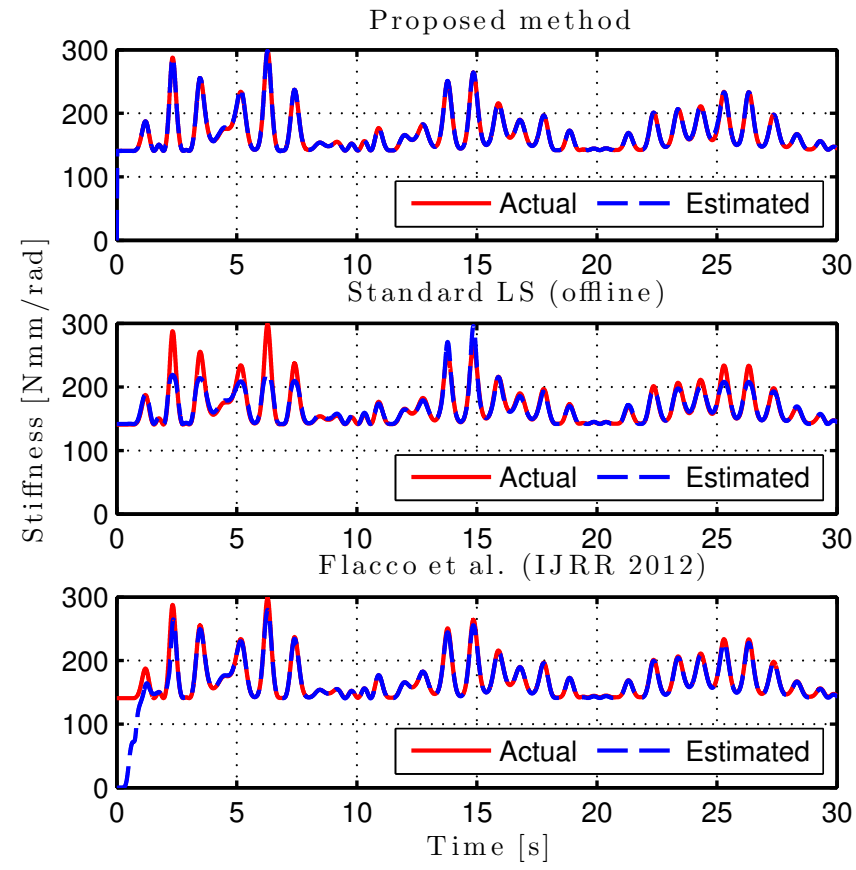

Fig. 2. Stiffness estimation for one of the two transmissions of the VSA-II, using three methods: Actual $\sigma$ (solid, red) and estimed $\hat{\sigma}$ (dashed, blue)

TABLE I

PERFORMANCE OF STIFFNESS ESTIMATION FOR THE VSA-II

\begin{tabular}{|c||c|c|c|}
\hline & Proposed & Standard LS & Flacco et al. [14] \\
\hline $\begin{array}{c}\mathrm{MSE} \\
{\left[\mathrm{N}^{2} \cdot \mathrm{mm}^{2} / \mathrm{rad}^{2}\right]}\end{array}$ & 0.58 & 102.64 & 31.60 \\
\hline $\begin{array}{c}\mathrm{MSREP} \\
{[\%]}\end{array}$ & 0.002 & 0.224 & 0.07 \\
\hline
\end{tabular}

To quantify the performance in stiffness estimation, we have considered two indices, namely the mean square error (MSE) and the (dimensionless) mean square relative error percentage (MSREP) over $k_{t o t}=k_{\max }-k_{\min }$ samples:

$$
\begin{aligned}
\mathrm{MSE} & =\frac{\sum_{k=k_{\min }}^{k_{\max }}\left[\left(\sigma_{k}-\hat{\sigma}_{k}\right)^{2}\right]}{k_{\max }-k_{\min }} \\
\mathrm{MSREP} & =\frac{\sum_{k=k_{\min }}^{k_{\max }}\left[\left(\frac{\sigma_{k}-\hat{\sigma}_{k}}{\sigma_{k}}\right)^{2}\right]}{k_{\max }-k_{\min }} \cdot 100 .
\end{aligned}
$$

The indices for the given simulations, with $k_{\min }=2000(2$ [s]) samples discarded to avoid the initial transient phase, are presented in Tab I.

\section{Dealing With Signal Noise}

The presence of noise on the input and output signals has to be considered in a realistic model. While for the 
torque input $\tau$ a white gaussian noise with zero mean can be assumed, noise on the measured outputs $q$ and $\theta$ will depend on the type of sensors at hand. For instance, white noise was assumed in [15], whereas noise due to quantization and discretization of encoders was considered in [14].

In [14], a Modified Kinematic Kalman Filter (MKKF) was used to filter the encoder quantization noise. The MKKF is a causal filter that outputs a smoothed version of the input signal and a good estimate of its first time derivative, when the signal-to-noise ratio (SNR) is adequate. On the other hand, the estimator of [15] based on operational calculus results in a series of non-causal FIR filters followed by a RLS fitting. After some analysis of such non-causal filtering methods, we realized that their success relies on the validity of two operative conditions:

1) the same non-causal action is applied to all signals used in the RLS algorithm;

2) the characteristics of the flexible transmissions are quasistatic (namely, should change very slowly).

The FIR action is applied to a moving window $W$ of data, and the resulting value is assigned as output to the center of this window. Thus, when working on line, the filtered value has a time delay of $T_{s} W / 2$. Despite this delay, a very effective filtering action is achieved thanks to the possibility of considering previous and successive data. Condition 1) implies that, by having the same time delay for all signals used in the polynomial fitting, the estimated parameter vector $\hat{\boldsymbol{\alpha}}$ will simply inherit the same time delay: namely, at step $k$ we would estimate $\boldsymbol{\alpha}_{k-\frac{W}{2}}$. From Condition 2), it follows that $\hat{\boldsymbol{\alpha}}_{k-\frac{W}{2}}$ is also a good estimate for $\boldsymbol{\alpha}_{k}$.

With the above in mind, we propose to introduce two separate filtering actions: non-causal filters, so as to obtain a robust estimation of the input/output signals to be used in the QR-RLS algorithm; and causal filters, in order to have a non-delayed smoothed version of $\phi$ to be used in eq. (17) for the estimation of the current stiffness.

The filtered transmission deformation $\hat{\phi}_{k}$ can be obtained by two KKF, on the measured motor position $\theta$ and on the measured link position $q$. Let $x$ be a generic angular position and $\dot{x}$ the associated angular velocity. In order to estimate $\boldsymbol{\psi}(k)=\boldsymbol{\psi}\left(t_{k}\right)=\left(\begin{array}{ll}x(k) \dot{x}(k)\end{array}\right)^{T}$ with a KKF, we define

$$
\begin{aligned}
& \boldsymbol{\psi}(k)=\left(\begin{array}{cc}
1 & T_{s} \\
0 & 1
\end{array}\right) \boldsymbol{\psi}(k-1)+\boldsymbol{\mu}(k) \\
& z(k)=\left(\begin{array}{ll}
1 & 0
\end{array}\right) \boldsymbol{\psi}(k)+\nu(k),
\end{aligned}
$$

where $z(k)$ is the noisy sampled measure (the encoder angle in our case), and $\boldsymbol{\mu}(k)$ and $\nu(k)$ are discrete-time realizations of zero mean Gaussian noises having, respectively, covariance matrix $Q$ and variance $R$. In the state equation (28), acceleration is not considered and $\boldsymbol{\mu}$ represents also the noise due to this absence. By defining $\boldsymbol{\Gamma}=\left(T_{s}^{2} / 2 T_{s}\right)^{T}$, the covariance matrix of $\boldsymbol{\mu}$ is $\boldsymbol{Q}=V_{a} \boldsymbol{\Gamma} \boldsymbol{\Gamma}^{T}$, where $V_{a}$ is the variance associated to the state. While the variance $R$ of the measures is usually set to a constant value when the noise is Gaussian, in the case of encoder quantization the Modified KKF proposed in [14] shoudl be used instead.
A Savitzky-Golay (SG) filter [21] is applied to the data needed as inputs to the QR-RLS algorithm, namely $q, \theta$, and $\tau$. This digital filter is applied to a moving window of measured data and is able to obtain their smoothing, by increasing the SNR without largely distorting the signal. This is achieved by fitting successive sub-sets of adjacent data with a low-degree polynomial. When the data points are equally spaced, as in our case, an analytical solution to the least squares fitting can be found, in the form of a single set of coefficients that is applied to all data sub-sets. The filter output provides smoothed estimates of the input signal (and derivatives of the smoothed signal) at the central point of each data window. The QR-RLS applied on SG filtered data results in a very robust estimated vector $\hat{\boldsymbol{\alpha}}_{k-\frac{W}{2}}$.

Under Conditions 1) and 2), the stiffness estimation is obtained by putting together the two filtered parts, i.e.,

$$
\sigma_{k} \simeq \sum_{i=1}^{m} \hat{\alpha}_{i, k-\frac{W}{2}} g_{i}\left(\hat{\phi}_{k}\right)
$$

\section{Results With ReAlistic Signals}

To show the effectiveness of the proposed approach and its robustness with respect to signal noise we have compares it with two state-of-the-art stiffness estimators.

The first one is the residual-based stiffness estimator in [14]. We have simulated the same VSA-II model under exactly the same design and operative conditions: encoder quantization, white noise on motor torques, torque input profiles, polynomials used for the fitting, etc. The same causal MKKF used in [14] was applied for the estimate $\hat{\phi}$, and we used a SG filter with a window of 1 second ( $W=1000$ samples at $T_{s}=0.001[\mathrm{~s}]$ ) and a 20-th degree polynomial for estimating the QR-RLS inputs. The forgetting factor was not considered $(\lambda=1)$.

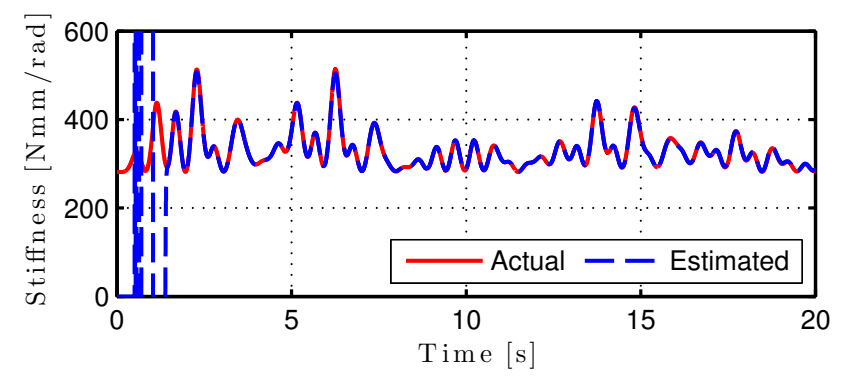

Fig. 3. Estimation of the total stiffness of the VSA-II device, when modeled and controlled under the same realistic conditions as in [14]: Actual $\sigma$ (solid, red) and estimated $\hat{\sigma}$ (dashed, blue)

The total stiffness obtained with the proposed method shows a very good quality of the estimation, as apparent in Fig. 3. The result is similar to the one in [14], as indicated by the numerical comparison of indices. We obtained here $M S E=2.0919\left[\mathrm{~N}^{2} \cdot \mathrm{mm}^{2} / \mathrm{rad}^{2}\right]$ and $M S R E P=0.5162 \%$, while in [14] we had $M S E=92.2\left[\mathrm{~N}^{2} \cdot \mathrm{mm}^{2} / \mathrm{rad}^{2}\right]$ and $M S R E P=0.046 \%$. However, the present method provides in addition also a good estimate of the a priori unknown 
motor parameters: $\hat{B}_{1}=7.2360, \hat{B}_{2}=7.3022[\mathrm{Kg} \cdot \mathrm{m} \cdot \mathrm{mm}]$, and $\hat{D}_{\theta, 1}=0.9731, \hat{D}_{\theta, 2}=0.9980[\mathrm{~N} \cdot \mathrm{mm} \cdot \mathrm{s} / \mathrm{rad}]$.

The second work chosen for comparison is [15], where an antagonistic VSA with cubic flexibility torques has been considered. Also in this case, we simulated the same model $\left(B=10^{-4}\left[\mathrm{~N} \cdot \mathrm{m}^{2}\right], D_{\theta}=1.27[\mathrm{~N} \cdot \mathrm{m} \cdot \mathrm{s} / \mathrm{rad}]\right.$, and so on $)$, using the same fitting polynomials and torque inputs, and under the same operative conditions (heavy white noise on all signals). We used the KKF to estimate $\hat{\phi}$ with $V_{a}=10^{10}$ and $R=10^{4}$, and a SG filter with a window of 1 second $(W=$ 1000) and a polynomial of the 20-th degree for estimating the QR-RLS inputs. No forgetting factor was used as before.

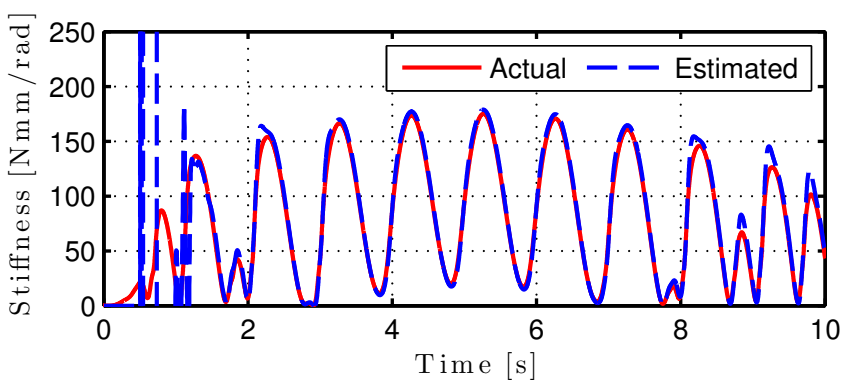

Fig. 4. Stiffness estimation for the antagonistic VSA device with cubic flexibility torques considered in [15]: Actual $\sigma$ (solid, red) and estimated $\hat{\sigma}$ (dashed, blue)

Estimation of the total stiffness is shown in Fig. 4. The present method gives $M S E=1.2583\left[\mathrm{~N}^{2} \cdot \mathrm{m}^{2} / \mathrm{rad}^{2}\right]$ and $M S R E P=1.6936 \%$, while in [15] the values were $M S E=4.2\left[\mathrm{~N}^{2} \cdot \mathrm{m}^{2} / \mathrm{rad}^{2}\right]$ and $M S R E P=0.7 \%$. Thus, a similarly good performance is achieved. Indeed, our method is also able to estimate the a priori unknown motor parameters: $\hat{B}_{1}=0.0023, \hat{B}_{2}=7.1942 \times 10^{-4}\left[\mathrm{~N} \cdot \mathrm{m}^{2}\right]$, and $\hat{D}_{\theta, 1}=1.2843, \hat{D}_{\theta, 2}=1.2706[\mathrm{~N} \cdot \mathrm{m} \cdot \mathrm{s} / \mathrm{rad}]$.

It is difficult to assess in general the superiority of one method over the other in terms of performance, especially when just looking at a single or few simulations. Nonetheless, we can at least conclude that the present method has the same stiffness estimation quality of state-of-the-art algorithms, while it does not rely on the knowledge of motor parameters (actually, of any physical parameter).

\section{CONCLUSIONS}

Building on our previous results, we have presented an improved method for the on-line estimation of stiffness in flexible transmissions and VSA devices that uses only on input-output signals, without requiring knowledge of motor parameters (which are also estimated as a by-product) or needing a joint torque sensor. Robustness to poor excitation conditions and to noisy signals is obtained thanks to the use of a QR decomposition in the Recursive Least Squares fitting method, and by suitably processing signals with a combination of causal and non-causal filters.

We are currently planning an experimental validation of this stiffness estimation method, and a comparison with other existing algorithms, on a benchmark VSA device, the low-cost modular qbmove developed by the Natural Motion
Initiative (a spin-off of the University of Pisa) within the EU SAPHARI project.

\section{REFERENCES}

[1] A. De Luca, F. Flacco, A. Bicchi, and R. Schiavi, "Nonlinear decoupled motion-stiffness control and collision detection/reaction for the VSA-II variable stiffness device," in Proc. IEEE/RSJ Int. Conf. on Intelligent Robots and Systems, 2009, pp. 5487-5494.

[2] R. Schiavi, G. Grioli, S. Sen, and A. Bicchi, "VSA-II: A novel prototype of variable stiffness actuator for safe and performing robots interacting with humans," in Proc. IEEE Int. Conf. on Robotics and Automation, 2008, pp. 2171-2176.

[3] M. Garabini, A. Passaglia, F. Belo, P. Salaris, and A. Bicchi, "Optimality principles in variable stiffness control: The VSA hammer," in Proc. IEEE/RSJ Int. Conf. on Intelligent Robots and Systems, 2011, pp. 3770-3775.

[4] S. Haddadin, T. Laue, U. Frese, S. Wolf, A. Albu-Schäffer, and G. Hirzinger, "Kick it with elasticity: Safety and performance in human robot soccer," Robotics and Autonomous Systems, vol. 57, pp. 761-775, 2009.

[5] S. Haddadin, M. Can Ozparpucu, and A. Albu-Schaffer, "Optimal control for maximizing potential energy in a variable stiffness joint," in Proc. 51th IEEE Conf. on Decision and Control, 2012, pp. 11991206.

[6] D. J. Braun, F. Petit, F. Huber, S. Haddadin, P. van der Smagt, A. AlbuSchaffer, and S. Vijayakumar, "Optimal torque and stiffness control in compliantly actuated robots," in Proc. IEEE/RSJ Int. Conf. on Intelligent Robots and Systems, 2012, pp. 2801-2808.

[7] G. Grioli and A. Bicchi, "A non-invasive real-time method for measuring variable stiffness," in Proc. Conf. Robotics: Science and Systems (RSS 2010), Zaragoza, E, June 2010.

[8] A. Serio, G. Grioli, I. Sardellitti, N. G. Tsagarakis, and A. Bicchi, "A decoupled impedance observer for a variable stiffness robot," in Proc. IEEE Int. Conf. on Robotics and Automation, 2011, pp. 5548-5553.

[9] G. Grioli and A. Bicchi, "A real-time parametric stiffness observer for VSA devices," in Proc. IEEE Int. Conf. on Robotics and Automation, 2011, pp. 5535-5540.

[10] F. Flacco and A. De Luca, "Residual-based stiffness estimation in robots with flexible transmissions," in Proc. IEEE Int. Conf. on Robotics and Automation, 2011, pp. 5541-5547.

[11] A. Cirillo, G. De Maria, C. Natale, and S. Pirozzi, "A mechatronic approach for robust stiffness estimation of variable stiffness actuators," in Proc. IEEE/ASME Int. Conf. on Advanced Intelligent Mechatronics, 2013, pp. 399-404.

[12] F. Flacco and A. De Luca, "Stiffness estimation and nonlinear control of robots with variable stiffness actuation," in 18th IFAC World Congr, 2011, pp. 6872-6879.

[13] F. Flacco, A. De Luca, I. Sardellitti, and N. G. Tsagarakis, "Robust estimation of variable stiffness in flexible joints," in Proc. IEEE/RSJ Int. Conf. on Intelligent Robots and Systems, 2011, pp. 4026-4033.

[14] F. Flacco, A. De Luca, I. Sardellitti, and N. Tsagarakis, "On-line estimation of variable stiffness in flexible robot joints," Int. J. of Robotics Research, vol. 31, no. 13, pp. 1556-1577, 2012.

[15] T. Ménard, G. Grioli, and A. Bicchi, "A real time robust observer for an agonist-antagonist variable stiffness actuator," in Proc. IEEE Int. Conf. on Robotics and Automation, 2013, pp. 3988-3993.

[16] A. Bicchi, M. Bavaro, G. Boccadamo, D. De Carli, R. Filippini, G. Grioli, M. Piccigallo, A. Rosi, R. Schiavi, S. Sen, and G. Tonietti, "Physical Human-Robot Interaction: Dependability, safety, and performance," in Proc. 10th Int. Work. on Advanced Motion Control, 2008, pp. 9-14.

[17] R. Van Ham, T. Sugar, B. Vanderborght, K. Hollander, and D. Lefeber, "Compliant actuator designs," IEEE Robotics and Automation Mag., vol. 16, no. 3, pp. 81-94, 2009.

[18] S. Migliore, E. Brown, and S. DeWeerth, "Biologically inspired joint stiffness control," in Proc. IEEE Int. Conf. on Robotics and Automation, 2005, pp. 4508-4513.

[19] J. A. Apolinario Jr., Ed., QRD-RLS Adaptive Filtering. Springer, 2009.

[20] J. G. McWhirter, "Recursive least-squares minimization using a systolic array," in Proc. of SPIE, Real Time Signal Processing V, 1983, pp. $105-112$.

[21] A. Savitzky and M. J. E. Golay, "Smoothing and differentiation of data by simplified least squares procedures," Analytical Chemistry, vol. 36, no. 8, pp. 1627-1639, 1964. 